OCHA-PP-130

AJC-HEP-32

December 16, 1999

Revised January 30, 2000

\title{
Production of Scalar Higgs and Pseudoscalar Higgs in Multi-Higgs Doublet Models at $\gamma \gamma$ Colliders
}

\author{
Eri AsAKAWA, \\ Graduate School of Humanities and Sciences, Ochanomizu University \\ 1-1 Otsuka 2-chome, Bunkyo, Tokyo 112-8610, JAPAN \\ e-mail: g9870401@edu.cc.ocha.ac.jp \\ Jun-ichi Kamoshita and Akio Sugamoto \\ Department of Physics, Ochanomizu University \\ 1-1 Otsuka 2-chome, Bunkyo, Tokyo 112-8610, JAPAN \\ and \\ Isamu WATANABE \\ Akita Keizaihoka University Junior College \\ 46-1 Morisawa, Sakura, Shimokita-te, Akita 010-8515, JAPAN \\ e-mail: isamu@akeihou-u.ac.jp
}

\begin{abstract}
We present the effects of heavy CP-even $(H)$ and CP-odd $(A)$ Higgs bosons on the production cross section of the process $\gamma \gamma \rightarrow t \bar{t}$ at the energy around the mass poles of the Higgs bosons. It is found that the interference between $H$ and $A$ with the small mass gap, as well as the ones between Higgs bosons and the continuum, contributes to the cross section, if the photon beams are polarized and if we observe the helicity of the top quarks. It is demonstrated in the framework of the minimal supersymmetric extension of the standard model that the $H$ and $A$ contributions can be sizable at future $\gamma \gamma$ colliders for small value of $\tan \beta$. The methods to measure the CP-parity of the Higgs boson are also presented. The statistical significances of detecting the Higgs signals and measuring the Higgs CP-parity are evaluated.
\end{abstract}




\section{Introduction}

Search for the Higgs bosons and the precise measurements on their properties, such as the masses, the decay widths and the decay branching ratios, are the most important subjects to study the mechanism of the electroweak symmetry breaking. In the standard model (SM) of the particle physics, only one physical neutral Higgs boson appears. On the other hand, models with multiple Higgs doublets have CP-even and odd neutral Higgs bosons and charged Higgs bosons, if CP is a good symmetry.

It is of great interest to examine how we are able to observe these Higgs bosons, and how their signals look like. A $\gamma \gamma$ option [1] of the future linear $e^{+} e^{-}$colliders gives an ideal place to look for such Higgs signals [2]. Neutral Higgs bosons can be produced via loop diagrams of charged particles. In the SM case, if the Higgs boson is lighter than about $140 \mathrm{GeV}$, it can be detected and its two-photon decay width can be measured accurately by looking for the $b \bar{b}$ pair decay mode of the Higgs boson [3]. When the Higgs boson is heavy in the SM, the process $\gamma \gamma \rightarrow Z Z$ is useful to detect its signal [4], since there is no tree-level background in this process.

It is also expected that in the extended models of the Higgs sector, the detection of a light Higgs boson may be performed just like in the SM, as far as it decays dominantly into a $b \bar{b}$ pair. On the other hand, it may be ineffective to look for a heavy CP-even Higgs boson $(H)$ in the $Z Z$ decay mode, since the branching fraction may be suppressed as often happens in the supersymmetric extensions of the SM. Furthermore, a CP-odd Higgs boson $(A)$ does not have the $Z Z$ decay mode. Then, the $t \bar{t}$ decay mode can be used, if the Higgs bosons in interest are sufficiently heavy and have substantial branching fractions for the $t \bar{t}$ decay. In this case, both $H$ and $A$ bosons may contribute to the process $\gamma \gamma \rightarrow t \bar{t}$ around their mass poles.

It is also notable that $H$ and $A$ bosons acquire masses with similar magnitudes for some appropriate values of parameters in the Higgs sector. The amplitudes of $\gamma \gamma \rightarrow$ $H \rightarrow t \bar{t}$ and $\gamma \gamma \rightarrow A \rightarrow t \bar{t}$ can interfere, if the mass difference between $H$ and $A$ bosons is smaller than or in the same order of the decay widths of these bosons, and if the helicities of the initial and final particles are properly selected. Additional interferences between these Higgs production amplitudes and the continuum one should also be taken into account, if the resonant and the continuum amplitudes have comparable magnitudes near the resonance. This actually occurs in the case of heavy Higgs bosons, since the total decay widths in this case are large enough to reduce the peak magnitudes of the Higgs production amplitudes near the mass poles.

In this paper, we study production and decay of heavy Higgs bosons of both CPparity in the process $\gamma \gamma \rightarrow t \bar{t}$. We also discuss the feasibility of detecting heavy Higgs bosons at future $\gamma \gamma$ colliders by using the minimal supersymmetric extension of the SM (MSSM) as an example. We pay special attention to the interference effects between the variously contributing helicity amplitudes in the process. Focusing on the interference effects we propose a new method to measure the CP-parity of Higgs bosons.

The organization of this paper is as follows. In the next section, we study the interference of $H$ and $A$ bosons in the process $\gamma \gamma \rightarrow t \bar{t}$. The helicity amplitudes are calculated in section 3 , and the numerical results are given in section 4 . We give the conclusions in the last section. 
Preliminary results of this work have been reported at the "LCWS99" [5].

\section{Interference between Higgs-exchange Amplitudes}

One may naively expect that the interference between the $H$-exchange and the $A$-exchange amplitudes must vanish since $H$ and $A$ bosons have opposite $\mathrm{CP}$ parities. In fact, the top-loop amplitude that connects $H$ and $A$ bosons (Fig. 1) can easily be evaluated to be zero by using the trace technique of undergraduate exercises:

$$
\operatorname{Tr}\left[\gamma_{5}\left(\not p_{t}+m_{t}\right)\left(-\not p_{\bar{t}}+m_{t}\right)\right]=0
$$

Here we expressed the four-momenta of top and anti-top quarks as $p_{t}$ and $p_{\bar{t}}$, respectively. Taking the trace of the top-loop means summing up all the different helicities of the $t$ and $\bar{t}$. We can evade this summation, however, by selecting helicity states of the decaying $t$ and $\bar{t}$ from $H$ and $A$, since the helicity of a top quark can be determined statistically due to the decay angle dependence of $t \rightarrow b W^{+}[6]$, that is, we can measure the helicities of $t$ and $\bar{t}$ because their decays into $b$ and $W$ are parity-violating. It is in contrast to the production of lighter quarks which mostly form the low-lying pseudoscalar mesons.

Denoting the helicities of $t$ and $\bar{t}$ as $\lambda$ and $\bar{\lambda}$, respectively in the $t \bar{t}$ c.m. frame, there are two ways of forming a $t \bar{t}$ system from a decay of a spin-zero state $\langle\lambda \bar{\lambda}| ;$ i.e., $\lambda$ $=\bar{\lambda}=L(\langle L L|)$ and $\lambda=\bar{\lambda}=R(\langle R R|)$. The CP transformation CP interchanges $t_{L}$ and $\bar{t}_{R}$, and $t_{R}$ and $\bar{t}_{L}$, with an additional minus sign due to the parity difference between the particle and the anti-particle states:

$$
\begin{aligned}
\mathrm{CP}\langle L L| & =-\langle R R|, \\
\mathrm{CP}\langle R R| & =-\langle L L| .
\end{aligned}
$$

The CP eigenstates are $45^{\circ}$ mixtures of $\langle L L|$ and $\langle R R|$ :

$$
\begin{aligned}
& \mathrm{CP}(\langle L L|-\langle R R|)=+(\langle L L|-\langle R R|), \\
& \mathrm{CP}(\langle L L|+\langle R R|)=-(\langle L L|+\langle R R|) .
\end{aligned}
$$

The state (3a) couples to the CP-even boson $H$, and the state (3b) couples to the CP-odd boson $A$. Therefore, the states $\langle L L|$ and $\langle R R|$ are relevant to the difference and the sum of the $A$-exchange and the $H$-exchange amplitudes, respectively.

A similar argument can be repeated to the production amplitudes of $\gamma \gamma \rightarrow H / A$. In the frame of the linear polarization of initial photons, $H$ and $A$ states can be produced from collisions of the parallel and the perpendicularly polarized photons, respectively [7]. Though they are the CP eigenstates, the helicity eigenstates $|++\rangle$ and $|--\rangle$ are mixtures of them:

$$
\begin{aligned}
& |++\rangle=[(|x x\rangle-|y y\rangle)+i(|x y\rangle+|y x\rangle)] / 2, \\
& |--\rangle=[(|x x\rangle-|y y\rangle)-i(|x y\rangle+|y x\rangle)] / 2
\end{aligned}
$$

where we express the helicities of two photons in the kets in the left-hand-side of the equations, while the orientations of the linear polarizations of the photons are given in 
the right-hand-side when the momenta of the photons are along the $z$-axis direction. Then, we can expect the $H-A$ interference with the circularly polarized initial photons.

A $\gamma \gamma$ collider option [1,2] of the future linear colliders is an ideal place to look for the above interference in the process $\gamma \gamma \rightarrow H / A \rightarrow t \bar{t}$, since the circular polarization of the photon beams are naturally realized at the $\gamma \gamma$ colliders.

\section{$3 \quad$ Helicity Amplitudes}

As an example of the Higgs sector with multiple doublets, we study the MSSM having two Higgs doublets. There, we are able to demonstrate the definite numerical estimations with fixing a few MSSM parameters, without loosing the generality and the essence which are applicable for more complicated models. We have three neutral Higgs boson states in the MSSM, the light Higgs $h$, the heavy Higgs $H$ and the pseudoscalar Higgs $A$. The former two are CP-even, and the last one is CP-odd. As in the literature, we parameterize the Higgs sector by two parameters, the mass of $A, m_{A}$, and the ratio of the vacuum expectation values of two Higgs doublets, $\tan \beta$. In the MSSM, if $A$ is lighter than the $Z$ boson, the mass of $h, m_{h}$, is similar to $m_{A}$. On the other hand, as $m_{A}$ becomes heavier, the mass of $H, m_{H}$ approaches to $m_{A}$, while $m_{h}$ remains below $150 \mathrm{GeV}$. It is also noted that the current experimental lower bounds of $m_{A}$ and $m_{h}$ are $84.5 \mathrm{GeV}$ and $84.3 \mathrm{GeV}$, respectively [9].

Assuming $m_{A} \gg m_{Z}$, a possibility arises for observing the interference between $H$ and $A$ bosons. The process to be focused on is $\gamma \gamma \rightarrow t \bar{t}$ with the fixed helicities of $t$ and $\bar{t}$, and with the circular polarization of the initial photons. Both $H$ and $A$ bosons can contribute to the amplitudes around their mass poles as well as the continuum amplitudes (Fig. 2). We denote these amplitudes separately as,

$$
\mathcal{M}_{\gamma \gamma \rightarrow t \bar{t}}^{\Lambda \bar{\Lambda} \lambda \bar{\lambda}} \simeq \mathcal{M}_{H}^{\Lambda \bar{\Lambda} \lambda \bar{\lambda}}+\mathcal{M}_{A}^{\Lambda \bar{\Lambda} \lambda \bar{\lambda}}+\mathcal{M}_{\text {tree }}^{\Lambda \bar{\Lambda} \lambda \bar{\lambda}}
$$

where the subscripts $H, A$ and 'tree' mean the $H$-exchange, the $A$-exchange and the tree diagrams, respectively. The superscripts $\Lambda$ and $\bar{\Lambda}$ denote the initial photon helicities, $\lambda$ and $\bar{\lambda}$ denote $t$ and $\bar{t}$ helicities, respectively.

The Higgs-exchange amplitudes $\mathcal{M}_{H}^{\Lambda \bar{\Lambda} \lambda \bar{\lambda}}$ and $\mathcal{M}_{A}^{\Lambda \bar{\Lambda} \lambda \bar{\lambda}}$ are then given by the simple multiplication of the Higgs- $\gamma \gamma$ vertex function $\mathcal{A}_{H, A}^{\Lambda \bar{\Lambda}}$, the Higgs propagator $\mathcal{B}_{H, A}$ and the decay part $\mathcal{C}_{H, A}^{\lambda \bar{\lambda}}$ of $H, A \rightarrow t \bar{t}$ :

$$
\begin{aligned}
& \mathcal{M}_{H}^{\Lambda \bar{\Lambda} \lambda \bar{\lambda}}=\mathcal{A}_{H}^{\Lambda \bar{\Lambda}} \mathcal{B}_{H} \mathcal{C}_{H}^{\lambda \bar{\lambda}} \\
& \mathcal{M}_{A}^{\Lambda \bar{\Lambda} \lambda \bar{\lambda}}=\mathcal{A}_{A}^{\Lambda \bar{\Lambda}} \mathcal{B}_{A} \mathcal{C}_{A}^{\lambda \bar{\lambda}} .
\end{aligned}
$$

\subsection{Higgs-Photon Couplings}

In the one-loop approximation, $W$ bosons, quarks, charged leptons, squarks, charged sleptons, charged Higgs bosons and charginos contribute to the $H \gamma \gamma$ vertex function 
$\mathcal{A}_{H}^{\Lambda \bar{\Lambda}}$. Only quarks, charged leptons and charginos contribute to $\mathcal{A}_{A}^{\Lambda \bar{\Lambda}}$. The dominant contributors are the top quark and the $W$ boson (only in the $H$ case) for small values of $\tan \beta$, if their superpartners are heavy enough comparing with Higgs bosons. The absolute values of $\mathcal{A}_{H, A}^{\Lambda \bar{\Lambda}}$ can be derived from the formulae of the Higgs partial decay widths into two photons $\Gamma(H, A \rightarrow \gamma \gamma)$, which are found in the literature [8]. It is essential to know not only the absolute values but also the relative phases of these amplitudes, since we wish to compute the interference. The formulae of $\Gamma(H, A \rightarrow \gamma \gamma)$ in the literature keep the relative phases of the relevant loops only in each amplitude of $H$ and $A$ bosons, but the relative phases between them are missing, since only the absolute values of the respective amplitudes have been necessary to obtain the corresponding decay widths. Therefore, we have calculated the relative phase of the $H$-exchange and the $A$-exchange amplitudes by using the top-quark contribution. We have found that $\mathcal{A}_{A}^{\Lambda \bar{\Lambda}}$ changes its sign according to the helicities of the initial photons, while $\mathcal{A}_{H}^{\Lambda \bar{\Lambda}}$ is independent of the photons helicities:

$$
\begin{array}{ll}
\mathcal{A}_{H}^{\Lambda \bar{\Lambda}}=-\frac{\alpha_{\mathrm{QED}} g}{8 \pi} \frac{m_{H}^{2}}{m_{W}} \sum_{i} I_{H}^{i} & (\text { for } \Lambda \bar{\Lambda}= \pm \pm), \\
\mathcal{A}_{A}^{\Lambda \bar{\Lambda}}= \pm i \frac{\alpha_{\mathrm{QED}} g}{8 \pi} \frac{m_{A}^{2}}{m_{W}} \sum_{i} I_{A}^{i} & (\text { for } \Lambda \bar{\Lambda}= \pm \pm), \\
\mathcal{A}_{H}^{\Lambda \bar{\Lambda}}=\mathcal{A}_{A}^{\Lambda \bar{\Lambda}}=0 & (\text { for } \Lambda \bar{\Lambda}= \pm \mp),
\end{array}
$$

where $g$ is the weak coupling constant, $m_{W}$ is the $W$ boson mass and the functions $I_{H, A}^{i}$ can be found in eq. C.4 of Ref. 8. The photon-helicity dependent sign appears only in eq. (7b), which is consistent with our previous observation in eq. (田).

\subsection{Higgs Propagators}

It is instructive to study the energy dependence of the propagators $\mathcal{B}_{H, A}$ in the complex plane. A propagator with the decay width in its denominator draws a circle in the complex plane as its four-momentum squared $q^{2}$ increases:

$$
\mathcal{B} / i=\frac{i}{q^{2}-m^{2}+i m \Gamma}=\frac{1}{2 m \Gamma}\left[1+\exp \left(2 i \tan ^{-1} \frac{q^{2}-m^{2}}{m \Gamma}\right)\right],
$$

where $m$ and $\Gamma$ are the mass and the decay width of the propagating particle, respectively. The dominant energy dependence of the Higgs-exchange amplitudes $\mathcal{M}_{H, A}$ around the mass poles comes from this circular motion in the complex plane, if the energy is far from the thresholds of the loop particles.

\subsection{Higgs-Top Couplings}

The decay parts $\mathcal{C}_{H, A}^{\lambda \bar{\lambda}}$ are simply evaluated in the tree approximation:

$$
\begin{array}{ll}
\mathcal{C}_{H}^{\lambda \bar{\lambda}}=\mp \frac{g m_{t}}{m_{W}} \frac{\sin \alpha}{\sin \beta} E_{t} \beta_{t} & \left(\text { for } \lambda \bar{\lambda}={ }_{L L}^{R R}\right), \\
\mathcal{C}_{A}^{\lambda \bar{\lambda}}=-i \frac{g m_{t}}{m_{W}} \cot \beta E_{t} & \left(\text { for } \lambda \bar{\lambda}={ }_{L L}^{R R}\right), \\
\mathcal{C}_{H}^{\lambda \bar{\lambda}}=\mathcal{C}_{A}^{\lambda \bar{\lambda}}=0 & \left(\text { for } \lambda \bar{\lambda}={ }_{L R}^{R L}\right),
\end{array}
$$


where $\alpha$ is the mixing angle of the two neutral CP-even Higgs bosons, $E_{t}$ and $\beta_{t}$ are the energy and the velocity of the decaying top quark in the center of mass frame, respectively. Note here that the top-quark-helicity dependent sign appears only in the $H$ amplitude of eq. (9a), while it does not appear in the $A$ amplitude of eq. (9b). They are consistent with our previous observation in eq. (3).

\subsection{Tree Amplitudes}

In Table 1, $\mathcal{M}_{\text {tree }}^{\Lambda \bar{\Lambda} \lambda \bar{\lambda}}$ for each helicity combinations of external particles are summarized. Here $Q_{t}$ and $\theta_{t}$ are the electric charge and the scattering angle of the top quark, respectively. The factor $m_{t} / E_{t}$ appearing in the amplitudes with $\lambda=\bar{\lambda}$ is a consequence of the helicity conservation law in the massless limit of the fermion. Note that the CP transformation interchanges $\Lambda$ and $-\Lambda, \bar{\Lambda}$ and $-\bar{\Lambda}, t_{R}$ and $\bar{t}_{L}, t_{L}$ and $\bar{t}_{R}$, and $\cos \theta_{t}$ and $-\cos \theta_{t}$, each other, with an additional overall minus sign: eg., $\mathcal{M}_{\text {tree }}^{++R R}=-\mathcal{M}_{\text {tree }}^{-L L}$. If the circular polarizations of the initial photon beams are purely tuned to $100 \%$, there are only $t_{R} \bar{t}_{R}$ and $t_{L} \bar{t}_{L}$ productions. In the case of $\Lambda=\bar{\Lambda}=+$, the absolute value of the tree amplitude of $t_{R} \bar{t}_{R}$ is much greater than that of $t_{L} \bar{t}_{L}$ at high energies, due to difference of the factor $1+\beta_{t}$ and $1-\beta_{t}$.

\section{Numerical Estimates}

For our numerical estimates, the following input parameters are adopted: $m_{A}=400 \mathrm{GeV}$, $m_{W}=80.41 \mathrm{GeV}, m_{t}=175.0 \mathrm{GeV}$, the bottom quark pole mass $m_{b}=4.6 \mathrm{GeV}$, the charm quark pole mass $m_{c}=1.4 \mathrm{GeV}$, the tau lepton mass $m_{\tau}=1.777 \mathrm{GeV}$, the weak mixing angle $\sin ^{2} \theta_{W}=0.2312$, the QCD coupling constant at the $Z$ boson mass scale $\alpha_{s}\left(m_{Z}\right)$ $=0.117$. Since we don't want an undesirable complication, we have chosen the sfermion mass scale $m_{\mathrm{SUSY}}=1 \mathrm{TeV}$, the $\mathrm{SU}(2)$ gaugino mass parameter $M_{2}=500 \mathrm{GeV}$ and the higgsino mixing mass parameter $\mu=-500 \mathrm{GeV}$, which result in heavy supersymmetric particles. Thus, in the two-photon decay amplitudes of the Higgs bosons, the contributions of the supersymmetric loops are suppressed. The masses, the decay widths and the decay branching ratios of the Higgs bosons are evaluated by the program HDECAY [10] with choosing a value of $\tan \beta$. With the above parameters and the value of $\tan \beta$ between 1 and 20, we found that no real production of the new particles other than $h, H$ and $A$ is allowed at the energy scale of $m_{A}$ or $m_{H}$ and the new particles do not give significant contributions to Higgs- $\gamma \gamma$ vertices.

\subsection{Breit-Wigner Approximation}

A significant interference between the $H$-exchange and the $A$-exchange amplitudes can be expected with the small mass difference and the large decay widths of these Higgs bosons. For $m_{A}=400 \mathrm{GeV}$, Fig. 3 shows that the mass splitting between $H$ and $A$ is small for $\tan \beta \sim 10$. The total decay widths of $H$ and $A\left(\Gamma_{H}\right.$ and $\left.\Gamma_{A}\right)$ are large for both $\tan \beta \sim 1$ and $\tan \beta \sim 10$, as are seen in Fig. 3 . 
On the other hand, the peak cross sections of $\gamma \gamma \rightarrow H / A \rightarrow t \bar{t}$ computed by the Breit-Wigner approximation give a first guess in the magnitude of the Higgs contributions against the continuum contribution:

$$
\sigma_{\mathrm{BW}}^{\mathrm{peak}}=16 \pi \operatorname{Br}(H, A \rightarrow \gamma \gamma) \operatorname{Br}(H, A \rightarrow t \bar{t}) / m_{H, A}^{2}
$$

where $B r$ 's denote the decay branching ratios of $H$ or $A$ boson. The Breit-Wigner peak cross sections of $H$ and $A$ are plotted in Fig. 4 . As one can see from the figure, $\tan \beta \gtrsim$ 10 results in suppression of these peak cross sections, which is due to the small branching ratios of both $H, A \rightarrow \gamma \gamma$ and $H, A \rightarrow t \bar{t}$. We have chosen $\tan \beta=3$ and 7 in our simulation below. The values of the masses, the total decay widths, the two-photon decay branching ratios and the $t \bar{t}$ branching ratios of $H$ and $A$ adopted in our further calculations are summarized in Table 2 .

\subsection{Cross Sections of $\gamma \gamma \rightarrow t \bar{t}$}

Fig. 5 shows the energy dependence of $\gamma_{+} \gamma_{+} \rightarrow t_{\lambda} \bar{t}_{\bar{\lambda}}$ cross section for $\tan \beta=3$ and 7 . The solid curves represent the cross sections with the interference; $\sigma^{++\lambda \bar{\lambda}}$, see eq. (11a), while the dashed curves show the one obtained by neglecting the interference; $\sigma_{0}^{++\lambda \bar{\lambda}}$, see eq. (11b). The cross sections without any Higgs production are also superimposed as

in the dot-dashed curves; $\sigma_{\text {tree }}^{++\lambda \bar{\lambda}}$, see eq. (11d). The manifest definitions of these cross sections are as follows:

$$
\begin{aligned}
\sigma^{++\lambda \bar{\lambda}} & =\frac{N_{c} \beta_{t}}{32 \pi s_{\gamma \gamma}} \int_{-1}^{+1} d \cos \theta_{t}\left|\mathcal{M}_{\gamma \gamma \rightarrow t \bar{t}}^{++\lambda \bar{\lambda}}\right|^{2} \\
\sigma_{0}^{++\lambda \bar{\lambda}} & =\frac{N_{c} \beta_{t}}{16 \pi s_{\gamma \gamma}}\left[\left|\mathcal{M}_{H}^{++\lambda \bar{\lambda}}\right|^{2}+\left|\mathcal{M}_{A}^{++\lambda \bar{\lambda}}\right|^{2}\right]+\sigma_{\text {tree }}^{++\lambda \bar{\lambda}} \\
\sigma_{\text {tree }}^{++\lambda \bar{\lambda}} & =\frac{N_{c} \beta_{t}}{32 \pi s_{\gamma \gamma}} \int_{-1}^{+1} d \cos \theta_{t}\left|\mathcal{M}_{\text {tree }}^{++\lambda \bar{\lambda}}\right|^{2}
\end{aligned}
$$

where $N_{c}$ is the color factor of the top quark and $s_{\gamma \gamma}$ is the collision energy squared. As one can see in the figures, the interference is constructive at the energy below $m_{A}$, while it is destructive above $m_{A}$. Especially, the cross sections for the $(++R R)$ case, $\sigma^{++R R}$, is strongly variant and have sharp troughs, which show us drastic effects of the interference.

As described above, the interference term consists of three distinct contributions; i.e., $A-H, H$-tree and $A$-tree. We define the following four quantities representing the interference contributions:

$$
\begin{aligned}
& \Delta \sigma^{++\lambda \bar{\lambda}}=\sigma^{++\lambda \bar{\lambda}}-\sigma_{0}^{++\lambda \bar{\lambda}}=\Delta \sigma_{A-H}^{++\lambda \bar{\lambda}}+\Delta \sigma_{H \text {-tree }}^{++\lambda \bar{\lambda}}+\Delta \sigma_{A \text {-tree }}^{++\lambda \bar{\lambda}} \\
& \Delta \sigma_{A-H}^{++\lambda \bar{\lambda}}=\frac{N_{c} \beta_{t}}{8 \pi s_{\gamma \gamma}} \Re e\left(\mathcal{M}_{A}^{++\lambda \bar{\lambda}} \mathcal{M}_{H}^{++\lambda \bar{\lambda} *}\right) \\
& \Delta \sigma_{H-\text { tree }}^{++\lambda \bar{\lambda}}=\frac{N_{c} \beta_{t}}{16 \pi s_{\gamma \gamma}} \int_{-1}^{+1} d \cos \theta_{t} \Re e\left(\mathcal{M}_{H}^{++\lambda \bar{\lambda}} \mathcal{M}_{\text {tree }}^{++\lambda \bar{\lambda} *}\right) \\
& \Delta \sigma_{A \text {-tree }}^{++\lambda \bar{\lambda}}=\frac{N_{c} \beta_{t}}{16 \pi s_{\gamma \gamma}} \int_{-1}^{+1} d \cos \theta_{t} \Re e\left(\mathcal{M}_{A}^{++\lambda \bar{\lambda}} \mathcal{M}_{\text {tree }}^{++\lambda \bar{\lambda} *}\right)
\end{aligned}
$$


These four values are plotted in Fig. 6. The $A$-tree term gives the dominant interference effect in the $(++\mathrm{LL})$ channel, whereas both the $A$-tree and $H$-tree terms are significant in the $(++\mathrm{RR})$ channel.

The sign of the dominant Higgs-tree components $\Delta \sigma_{A-\text { tree }}^{++\lambda \bar{\lambda}}$ and $\Delta \sigma_{H-\text { tree }}^{++\lambda \bar{\lambda}}$ is dependent on the complex phases of the $\sum_{i} I_{H, A}^{i}$ factor in eqs. (7a), (7b) and of the propagator $\mathcal{B}_{H, A}$ in eq. (8). The $\sqrt{s}_{\gamma \gamma}$ dependence of the signs of the interference contributions from the Higgs-tree components are summarized in Table 3 , for the cases adopted in our numerical simulation, where $-\pi / 2<\arg \sum_{i} I_{H}^{i}<\pi / 2$ and $\pi / 2<\arg \sum_{i} I_{A}^{i}<3 \pi / 2$. From this table, one can qualitatively understand the energy dependence of $\Delta \sigma^{++\lambda \bar{\lambda}}$ found in Fig. 6. 6 .

\subsection{Convoluted Cross Sections with $\gamma \gamma$ Luminosity}

Due to the spread collision energy of $\gamma \gamma$ colliders, the observed cross section is a convoluted one with the $\gamma \gamma$ luminosity. A detailed study on the possible luminosity and polarization distributions at future $\gamma \gamma$ colliders has been performed by the simulation program CAIN [11]. However, these parameters are dependent on the machine design of the colliders. Thus we have adopted an ideal situation of the beam conversion that the photon beam is generated by the tree-level formula of the Compton backward-scattering and the effect of the finite scattering angle is negligible [12],

$$
\begin{aligned}
D(y) & =\frac{D_{1}+P_{e} P_{L} D_{2}}{D_{3}+P_{e} P_{L} D_{4}}, \\
P(y) & =\frac{P_{e} P_{1}+P_{L} P_{2}}{D_{1}+P_{e} P_{L} D_{2}}, \\
D_{1} & =1-y+1 /(1-y)-4 r(1-r), \\
D_{2} & =-r x(2-y)(2 r-1), \\
D_{3} & =\left(1-4 / x-8 / x^{2}\right) \log (x+1)+1 / 2+8 / x^{2}-1 / 2(x+1)^{2}, \\
D_{4} & =(1+2 / x) \log (x+1)-5 / 2+1 /(x+1)-1 / 2(x+1)^{2}, \\
P_{1} & =r x\left(1+(1-y)(2 r-1)^{2},\right. \\
P_{2} & =-(1-y+1 /(1-y))(2 r-1),
\end{aligned}
$$

where $D(y)$ and $P(y)$ are the energy and the circular polarization distributions, $y$ the energy fraction of the obtained beam photon to the original beam electron, $x$ the squared ratio of the total energy of the Compton scattering in the center-of-mass system to the electron mass, $r=y / x(1-y)$, and $P_{e}$ and $P_{L}$ the polarizations of the original electron beam and the laser photon beam, respectively. Here we have adopted the optimum value of $x, 2+2 \sqrt{2}$ [罒, and assumed ideal polarizations of the original beams, i.e., $P_{e}=+1$ and $P_{L}=-1$ for both sides. The luminosity distribution of the colliding $\gamma \gamma$ in each photon helicities can be evaluated as follows:

$$
\frac{1}{\mathcal{L}} \frac{d \mathcal{L}^{\Lambda \bar{\Lambda}}}{d z}=\int_{\log z / z_{\max }}^{\log z_{\max }} d \eta 2 z D\left(z e^{\eta}\right) \frac{1+\Lambda P\left(z e^{\eta}\right)}{2} D\left(z e^{-\eta}\right) \frac{1+\bar{\Lambda} P\left(z e^{-\eta}\right)}{2},
$$


where $z=\sqrt{s}_{\gamma \gamma} / \sqrt{s}_{e e}, z_{\max }=x /(x+1)=2 \sqrt{2}-2$ is the maximum value of $z, \eta$ is the rapidity of the colliding $\gamma \gamma$ system in the laboratory frame, $\mathcal{L}$ is the total $\gamma \gamma$ luminosity円. Resulting $\gamma \gamma$ luminosities are illustrated in Fig. 7 separately for the combination of the beam photon helicities.

One can evaluate the observed cross section by convoluting the cross section of the bare process calculated in the previous subsection with the above luminosity distribution, and by taking account of smearing due to the resolution of the detector. The resulting 'effective' cross section $\sigma^{\lambda \bar{\lambda}}$ is expressed as,

$$
\begin{aligned}
\sigma^{\lambda \bar{\lambda}}\left(\sqrt{s}_{e e} ; E_{n}, E_{m}\right)= & \int_{E_{n}}^{E_{m}} d E_{\mathrm{vis}} \int_{2 m_{t}}^{z_{\max } \sqrt{s}_{e e}} d \sqrt{s}_{\gamma \gamma} \sum_{\Lambda \bar{\Lambda}} \frac{1}{\mathcal{L}} \frac{d \mathcal{L}^{\Lambda \bar{\Lambda}}}{d \sqrt{s}} \\
& \times \hat{\sigma}^{\Lambda \bar{\Lambda} \lambda \bar{\lambda}}\left(\sqrt{s}_{\gamma \gamma}\right) G\left(\sqrt{s}_{\gamma \gamma}-E_{\mathrm{vis}}, r\right)
\end{aligned}
$$

where $E_{\text {vis }}$ is the visible energy in the detector, $E_{n}$ and $E_{m}$ the minimum and the maximum visible energy cuts, $r$ is the detector resolution on measuring $\sqrt{s}_{\gamma \gamma}$ and $G$ is the probability distribution of the detector resolution smearing. Here after, the bare cross section $\sigma^{\Lambda \bar{\Lambda} \lambda \bar{\lambda}}$ in the previous subsection is denoted as $\hat{\sigma}^{\Lambda \bar{\Lambda} \lambda \bar{\lambda}}$, as in the right-hand-side of eq. (15). We have assumed the Gaussian distribution $G(x, y)=\exp \left(-x^{2} / 2 y^{2}\right) / \sqrt{2 \pi} y$, with the detector resolution $r / \sqrt{s}_{\gamma \gamma}=\sqrt{40 \%^{2} /\left(\sqrt{s}_{\gamma \gamma} / \mathrm{GeV}\right)+2 \%^{2}}$, which is the one for the standard hadron calorimeter in the JLC design [13]. In Fig. 8, the effective cross sections for various combinations of the $t \bar{t}$ helicities are drawn as functions of $\sqrt{s}_{e e}$. The cut energies adopted here are $E_{n}=0.76 \sqrt{s}_{e e}$ and $E_{m}=0.82 \sqrt{s}_{e e}$ in order to use the energy range of the differential luminosity, $d \mathcal{L} / d z$, where both colliding photon beams are well polarized to be almost $+100 \%$. For the case of $\tan \beta=3$, one can clearly observe the existence of the Higgs bosons at both $L L$ and $R R$ modes, in comparison with the continuum cross sections. The effects of the interference terms are also sizable even in the convoluted effective cross sections at $\tan \beta=3$. These effects are destructive at $\sqrt{s}_{e e}<498 \mathrm{GeV}$ for $\sigma^{R R}$ and $<491 \mathrm{GeV}$ for $\sigma^{L L}$, and are constructive above these collider energies. Especially, the constructive contribution of the interference terms in $\sigma^{R R}$ is remarkably large at the wide range of the $\sqrt{s}_{e e}$ above $\sim 500 \mathrm{GeV}$. Thus there is a possibility that one can identify two Higgs signals separately by a careful analysis scanning $\sqrt{s}_{e e}$. In the case of $\tan \beta=$ 7, unfortunately, the signals of $H$ and $A$ are almost smeared out, since the magnitudes of the Higgs-exchanging amplitudes are small and the mass gap of $A$ and $H$ is narrow. For both values of $\tan \beta$, contributions coming from continuum $t_{L} \bar{t}_{R}$ and $t_{R} \bar{t}_{L}$ are negligibly small due to the suppressed $\mathcal{L}^{+-}$and $\mathcal{L}^{-+}$luminosities between the visible energy cuts, $E_{n}$ and $E_{m}$.

\subsection{CP-parity Measurement of the Higgs Bosons}

One can extract information about the CP-parity of Higgs bosons by observing the interference effects on the cross sections with the top helicity fixed?, $\sigma^{R R}$ and $\sigma^{L L}$. Here

\footnotetext{
${ }^{1}$ In this approximation, the right-hand-side of eq. (14) integrated over $z$ from 0 to $z_{\max }$ and summed up all $\Lambda \bar{\Lambda}$ combinations is unity.

2 The statistical tachnique to measure the top helicity is discussed in the sub-subsection 4.5.2.
} 
we present a convenient method to estimate the CP-parity of the Higgs boson from the measurements of $\sigma^{R R}$ and $\sigma^{L L}$.

First, for simplicity, let us assume only one Higgs boson of $H$ or $A$. The cross section of the bare process $\gamma_{+} \gamma_{+} \rightarrow t_{\lambda} \bar{t}_{\bar{\lambda}}$ with a Higgs resonance, $\hat{\sigma}^{++\lambda \bar{\lambda}}$, deviates from the continuum one, $\hat{\sigma}_{\text {tree }}^{++\lambda \bar{\lambda}}$. We can define the deviation as,

$$
\begin{aligned}
\Delta \hat{\sigma}_{t}^{++\lambda \bar{\lambda}} & \equiv \hat{\sigma}^{++\lambda \bar{\lambda}}-\hat{\sigma}_{\text {tree }}^{++\lambda \bar{\lambda}}=\Delta \hat{\sigma}_{\mathrm{Higgs}}+\Delta \hat{\sigma}_{\text {Higgs-tree }}^{++\lambda \bar{\lambda}}, \\
\Delta \hat{\sigma}_{\mathrm{Higgs}} & =\frac{N_{c} \beta_{t}}{16 \pi s_{\gamma \gamma}}\left|\mathcal{M}_{\mathrm{Higgs}}^{++\lambda \bar{\lambda}}\right|^{2}, \\
\Delta \hat{\sigma}_{\mathrm{Higgs-tree}}^{++\lambda \bar{\lambda}} & =\frac{N_{c} \beta_{t}}{16 \pi s_{\gamma \gamma}} \Re e\left(\mathcal{M}_{\mathrm{Higgs}}^{++\lambda \bar{\lambda}}\right) \int_{-1}^{+1} d \cos \theta_{t} \mathcal{M}_{\text {tree }}^{++\lambda \bar{\lambda}},
\end{aligned}
$$

where eqs. (16b) and (16a) are the contributions of the Higgs-exchange amplitude squared and the interference. According to the discussion on the CP-parity of the Higgs bosons in Secs. 2 and 3, one can see that $\Delta \hat{\sigma}_{\text {Higgs-tree }}^{++R R}$ and $\Delta \hat{\sigma}_{\text {Higgs-tree }}^{++L L}$ have the same signs for a pseudoscalar $A$, while $\Delta \hat{\sigma}_{\text {Higgs-tree }}^{++R R}$ and $\Delta \hat{\sigma}_{\text {Higgs-tree }}^{++L L}$ have the opposite signs for a scalar $H$. Since the tree amplitudes satisfy an inequality,

$$
\left|\mathcal{M}_{\text {tree }}^{++L L}\right|<\left|\mathcal{M}_{\text {tree }}^{++R R}\right|
$$

then, one can derive,

$$
\left|\Delta \hat{\sigma}_{\text {Higgs-tree }}^{++L L}\right|<\left|\Delta \hat{\sigma}_{\text {Higgs-tree }}^{++R R}\right| .
$$

Since the interference term $\Delta \hat{\sigma}_{\text {Higgs-tree }}^{++\lambda \bar{\lambda}}$ is suppressed more mildly than the Higgs term $\Delta \hat{\sigma}_{\text {Higgs }}$ far away from the resonance, $\Delta \hat{\sigma}_{t}^{++\lambda \bar{\lambda}}$ is negative at one side of $\sqrt{s}_{\gamma \gamma} \ll m_{\mathrm{Higgs}}$ and $\sqrt{s}_{\gamma \gamma} \gg m_{\mathrm{Higgs}}$. At the $\sqrt{s}_{\gamma \gamma}$ where $\Delta \hat{\sigma}_{t}^{++L L}$ is negative, we can conclude the following inequalities of judgment:

$$
\Delta \hat{\sigma}_{t}^{++L L}<0 \Rightarrow\left\{\begin{array}{cl}
\Delta \hat{\sigma}_{t}^{++R R}>0 & \text { for } H \\
\Delta \hat{\sigma}_{t}^{++R R}<0 & \text { for } A
\end{array}\right.
$$

Therefore, we can distinguish the CP-parity of the Higgs boson.

Unfortunately, it is not trivial whether this CP-parity judgment method always works after convoluting with the $\gamma \gamma$ luminosity, because the broad luminosity distribution may smear out information about the sign of the interference term. If there are plural Higgs bosons with near values of masses, as are often happen in the MSSM, the judgment may be obscured. Actually, in our mumerical simulation in the previous subsection 4.3 , $\Delta \hat{\sigma}_{t}^{++L L}$ is negative only at $\sqrt{s}_{e e}>545$ and $514 \mathrm{GeV}$ for the $\tan \beta=3$ and 7 cases, respectively. And at the same energy range, $\Delta \hat{\sigma}_{t}^{++R R}$ is also negative for both cases, which means the existence of a pseudoscalar Higgs $A$. In these cases, the effect of the scalar Higgs $H$ is smaller than that of $A$, and one cannot find out the signal of $H$ existence by this method.

\subsection{Number Count Estimates}




\subsubsection{Higgs boson detection}

The existence of Higgs bosons can be verified by observing the excess of the total cross section due to the Higgs resonances over the continuum. The estimated number of events of the process $\gamma \gamma \rightarrow t \bar{t}, N(t \bar{t})$, is proportional to the integrated luminosity $\int d t \mathcal{L}$ and the detection efficiency $\epsilon$,

$$
N(t \bar{t})=\sigma \cdot \epsilon \int d t \mathcal{L},
$$

where,

$$
\begin{aligned}
\sigma & =\sigma^{R R}+\sigma^{L L}+\sigma^{R L+L R}, \\
\sigma^{R L+L R} & =\sigma^{R L}+\sigma^{L R} .
\end{aligned}
$$

The statistical error of the cross section $E(\sigma)$ is estimated to be $\sqrt{N(t \bar{t})} / \epsilon \int d t \mathcal{L}$, and hence the relative error of the total cross section, $\mathcal{E}(\sigma)$, is inversely proportional to $\sqrt{\epsilon \int d t \mathcal{L}}$ :

$$
\mathcal{E}(\sigma) \equiv \frac{E(\sigma)}{\sigma}=\frac{1}{\sqrt{N(t \bar{t})}}=\frac{1}{\sqrt{\sigma \cdot \epsilon \int d t \mathcal{L}}} .
$$

Since the detection efficiency $\epsilon$ is not studied well for $\gamma \gamma$ colliders, we do not assume its particular numerical value. Instead, we define $\hat{\mathcal{E}}(\sigma)$ which is the special value of $\mathcal{E}(\sigma)$ with $\epsilon \int d t \mathcal{L}=1 \mathrm{fb}^{-1}$. The values of the total cross section $\sigma$ and its statistical error for the nominal integrated luminosity $\hat{\mathcal{E}}(\sigma)$ are listed in Table 4 for nine particular values between $\sqrt{s}_{e e}=480$ and $560 \mathrm{GeV}$. The statistical error $\mathcal{E}(\sigma)$ is smaller than $10 \%$ for all the cases listed in Table 6 , if we assume $1 \mathrm{fb}^{-1}$ of $\epsilon \int d t \mathcal{L}$.

The existence of the Higgs signals can be studied by comparing the measured effective cross section $\sigma$ with the computed one without Higgs resonances, $\sigma_{\text {tree }}$, which is defined similarly to $\sigma$. The statistical significance of the deviation of $\sigma$ from $\sigma_{\text {tree }}$ can be evaluated as,

$$
\mathcal{S} \equiv \frac{\sigma-\sigma_{\text {tree }}}{E(\sigma)}=\frac{\sigma-\sigma_{\text {tree }}}{\sqrt{\sigma}} \sqrt{\epsilon \int d t \mathcal{L}},
$$

where the sign of $\mathcal{S}$ indicates the sign of deviation. Since $\mathcal{S}$ is proportional to $\sqrt{\epsilon \int d t \mathcal{L}}$, we define $\hat{\mathcal{S}}$ which is the special value of $\mathcal{S}$ with $1 \mathrm{fb}^{-1}$ of $\epsilon \int d t \mathcal{L}$. The values of $\sigma_{\text {tree }}$ and $\hat{\mathcal{S}}$ are also listed in Table 1 . As is seen in the table, the effective cross section $\sigma$ deviates from $\sigma_{\text {tree }}$ in the one-sigma level at $\sqrt{s}_{e e}=480-500$ and $530-540 \mathrm{GeV}$ for $\tan \beta=3$, even with $1 \mathrm{fb}^{-1}$ of $\epsilon \int d t \mathcal{L}$. Tuning $\sqrt{s}_{e e}=490 \mathrm{GeV}$, one can establish the existence of the Higgs resonances at the two-sigma level with $1.6 \mathrm{fb}^{-1}$. On the other hand, we need more than $40 \mathrm{fb}^{-1}$ at $\sqrt{s}_{e e}=520 \mathrm{GeV}$ in the case of $\tan \beta=7$.

\subsubsection{CP-parity measurement}

In order to observe the CP-parity of the Higgs boson by the method described in the subsection 4.4, we need to measure $\sigma^{R R}$ and $\sigma^{L L}$ separately. The measurement of the top helicity can be performed statistically by using the decay angle dependence of the 
polarized top quark. In the tree-level approximation with massless bottom quark, one can easily derive the decay angle dependence for each of $t_{R}$ and $t_{L}$ :

$$
\begin{aligned}
& \frac{1}{\Gamma_{t_{R}}} \frac{d \Gamma_{t_{R}}}{d \cos \theta_{b}}=\frac{1}{\Gamma_{\bar{t}_{L}}} \frac{d \Gamma_{\bar{t}_{L}}}{d \cos \theta_{b}}=\frac{1}{2}\left[1-\frac{m_{t}^{2}-2 m_{W}^{2}}{m_{t}^{2}+2 m_{W}^{2}} \cos \theta_{b}\right] \simeq 0.50-0.20 \cos \theta_{b}, \\
& \frac{1}{\Gamma_{t_{L}}} \frac{d \Gamma_{t_{L}}}{d \cos \theta_{b}}=\frac{1}{\Gamma_{\bar{t}_{R}}} \frac{d \Gamma_{\bar{t}_{R}}}{d \cos \theta_{b}}=\frac{1}{2}\left[1+\frac{m_{t}^{2}-2 m_{W}^{2}}{m_{t}^{2}+2 m_{W}^{2}} \cos \theta_{b}\right] \simeq 0.50+0.20 \cos \theta_{b}
\end{aligned}
$$

where $\cos \theta_{b}$ is the emission angle of the (anti-)bottom quark in the rest frame of the decaying (anti-)top quark with respect to the direction of the (anti-)top momentum in the $t \bar{t} \mathrm{c} . \mathrm{m}$. frame. This decay angle dependence of the top quark allows us a simple method to estimate the top helicity, without studying the subsequent $W$ boson decay topology. The fraction of the forward $\left(0<\cos \theta_{b}<1\right)$ decay of the right-handed top quark $r_{F}$ is 0.40 , while the backward $\left(-1<\cos \theta_{b}<0\right)$ fraction $r_{B}$ is 0.60 , where we neglect the effects of the higher order diagrams and the finite resolution.

An event with the top quark decaying forward and with the anti-top quark decaying backward is most likely to be a $t_{L} \bar{t}_{L}$ event because of the above decay distributions. We define here two effective cross sections with decay angle selections: i.e. $\sigma_{F}$ with the forward top and the backward anti-top, and $\sigma_{B}$ with the backward top and the forward anti-top. By neglecting the miss-identification probability, we obtain,

$$
\begin{aligned}
& \sigma_{F}=\sigma^{R R} r_{F}^{2}+\sigma^{L L} r_{B}^{2}+\sigma^{R L+L R} r_{F} r_{B}, \\
& \sigma_{B}=\sigma^{R R} r_{B}^{2}+\sigma^{L L} r_{F}^{2}+\sigma^{R L+L R} r_{F} r_{B} .
\end{aligned}
$$

In Table 5, $\sigma^{R R}, \sigma^{L L}, \sigma^{R L+L R}, \sigma_{F}$ and $\sigma_{B}$ are listed for nine values of $\sqrt{s}_{e e}$ and at $\tan \beta=$ 3 and 7. Since $\sigma^{R L+L R}$ is small enough, we can neglect this contribution and can evaluate the values of the intrinsic effective cross sections $\sigma^{R R}$ and $\sigma^{L L}$ from $\sigma_{F}$ and $\sigma_{B}$ as,

$$
\left(\begin{array}{c}
\sigma^{R R} \\
\sigma^{L L}
\end{array}\right) \simeq \frac{1}{r_{B}^{4}-r_{F}^{4}}\left(\begin{array}{cc}
-r_{F}^{2} & r_{B}^{2} \\
r_{B}^{2} & -r_{F}^{2}
\end{array}\right)\left(\begin{array}{c}
\sigma_{F} \\
\sigma_{B}
\end{array}\right)
$$

The validity of neglecting $\sigma^{R L+L R}$ is estimated, and the shift to the measured cross section by this approximation from the true value is found to be less than $0.4 \mathrm{fb}(\sim 4.2 \%)$ for the cases listed in Table 5 .

The relative errors of the $\sigma^{R R}$ and $\sigma^{L L}, \mathcal{E}\left(\sigma^{R R}\right)$ and $\mathcal{E}\left(\sigma^{L L}\right)$, are similarly defined as $\mathcal{E}(\sigma)$ in eq. (22), and can be evaluated as,

$$
\begin{aligned}
& \mathcal{E}\left(\sigma^{R R}\right)=\frac{\sqrt{\sigma_{F} r_{F}^{4}+\sigma_{B} r_{B}^{4}}}{\left|\sigma_{F} r_{F}^{2}-\sigma_{B} r_{B}^{2}\right|} \frac{1}{\sqrt{\epsilon^{\prime} \int d t \mathcal{L}}}, \\
& \mathcal{E}\left(\sigma^{L L}\right)=\frac{\sqrt{\sigma_{F} r_{B}^{4}+\sigma_{B} r_{F}^{4}}}{\left|\sigma_{F} r_{B}^{2}-\sigma_{B} r_{F}^{2}\right|} \frac{1}{\sqrt{\epsilon^{\prime} \int d t \mathcal{L}}},
\end{aligned}
$$

where $\epsilon^{\prime}$ is the detection efficiency for this observation. Since these relative errors are again proportional to $1 / \sqrt{\epsilon^{\prime} \int d t \mathcal{L}}$, we introduce the nominal relative errors with $1 \mathrm{fb}^{-1}$ of $\epsilon^{\prime} \int d t \mathcal{L}$, 
$\hat{\mathcal{E}}\left(\sigma^{R R}\right)$ and $\hat{\mathcal{E}}\left(\sigma^{L L}\right)$ which are the special values of $\mathcal{E}\left(\sigma^{R R}\right)$ and $\mathcal{E}\left(\sigma^{L L}\right)$, respectively, with $1 \mathrm{fb}^{-1}$ of $\epsilon^{\prime} \int d t \mathcal{L}$. Their estimated values are listed in Table 6 . According to the table, we find that $\sigma^{R R}$ is measurable within the accuracy of $10 \%$ if one accumulates $5.6 \mathrm{fb}^{-1}$ of $\epsilon^{\prime} \int d t \mathcal{L}$, while the statistical error exceeds $35 \%$ for $\sigma^{L L}$ with the same luminosity. To achieve a $10 \%$ accuracy for $\sigma^{L L}$, the desired $\epsilon^{\prime} \int d t \mathcal{L}$ is $68-490 \mathrm{fb}^{-1}$, that may be realized by a future technology of 'an ultimately high luminosity' [14].

The statistical significances of the deviation of $\sigma^{R R}$ and $\sigma^{L L}$ from the ones without any Higgs resonances are again defined similarly to eq. (23), and these values with 1 $\mathrm{fb}^{-1}, \hat{S}^{R R}$ and $\hat{S}^{L L}$, are also listed in Table 6. As was mentioned in the subsection 4.4, the proposed technique to determine CP-parity is not applicable efficiently for the multiHiggs bosons almost degenerated in the cases of our numerical estimates. Table 6 shows that the technique requires thousands $\mathrm{fb}^{-1}$ of the luminosity even in the $\tan \beta=3$ case.

\section{Conclusions}

We have presented in this paper the effects of heavy CP-even and CP-odd Higgs bosons on the production cross section of the process $\gamma \gamma \rightarrow t \bar{t}$ at the energy around the mass poles of the Higgs bosons. It has been found that the interference between $H$ and $A$ with the small mass gap, as well as the ones between Higgs bosons and the continuum, contributes to the cross section, if the photon beams are polarized and if we observe the helicity of the top quarks. It has been demonstrated in the framework of the MSSM that the $H$ and $A$ contributions can be sizable at future $\gamma \gamma$ colliders for small value of $\tan \beta$. The methods to evaluate the cross sections, to detect the Higgs bosons and to measure the CP-parity of the Higgs boson have also been presented. The statistical significances of detecting the Higgs signals and measuring the Higgs CP-parity have been evaluated. It has been found that the effective cross section can be measured within $10 \%$ of statistical accuracy with $1 \mathrm{fb}^{-1}$ of the integrated luminosity multiplied by the detection efficiency. The existence of the Higgs signals with $m_{A}=400 \mathrm{GeV}$ over the continuum cross section can be verified in the two-sigma level with $1.6 \mathrm{fb}^{-1}$ for the $\tan \beta=3$ case and $40 \mathrm{fb}^{-1}$ for the $\tan \beta=7$ case.

\section{Acknowledgements}

This work is supported in part by the Grant-in-Aid for Scientific Research (No. 11640262) and the Grant-in-Aid for Scientific Research on Priority Areas (No. 11127205) from the Ministry of Education, Science and Culture, Japan.

The authors would like to thank K. Hagiwara for valuable discussions and reading the manuscript. The authors would also like to thank T. Kon, T. Ohgaki, Y. Okada, T. Tauchi, T. Takahashi for useful discussions. 


\section{References}

1. I.F. Ginzburg, G.L. Serbo and V.I. Tel'nov, Pis'ma Zh. Eksp. Teor. Fiz., 34 (1981) 514 [JETP Lett., 34 (1982) 491]; Nucl. Instrum. Methods, 205 (1083) 47.

2. As a summary report, see ' $\gamma \gamma$ Collider as an Option of $J L C$ ', ed. I. Watanabe et al., KEK Report 97-17, KEK, March 1998.

3. T. Ohgaki, T. Takahashi and I. Watanabe, Phys. Rev., D56 (1997) 1723.

4. G. V. Jikia, Nucl. Phys., B405 (1993) 24.

M.S. Berger, Phys. Rev., D48 (1993) 5121.

5. E. Asakawa, to be published in the proceedings of "4th International Workshop on Linear Colliders (LCWS99)", Sitges, Barcelona, Spain, 28 Apr. - 5 May, 1999.

I. Watanabe, ibid.

6. K. Hagiwara, H. Murayama and I. Watanabe, Nucl. Phys. B367 (1991) 257.

7. C.N. Yang, Phys. Rev. 77 (1950) 242.

B. Grazadkowski and J.F. Gunion, Phys. Lett. B291 (1992) 361.

M. Krämer, J. Kühn, M.L. Stong and P.M. Zerwas, Z. Phys. C64 (1994) 21.

J.F. Gunion and J.G. Kelly, Phys. Lett. B333 (1994) 110.

S.Y. Choi and K. Hagiwara, Phys. Lett., B359 (1995) 369.

8. See, J.F. Gunion, H.E. Haber, G. Kane and S. Dawson, 'Higgs Hunter's Guide', AddisonWesley Publishing Company, (1990), and references therein.

9. P. McNamara, The LEP Higgs Working Group, LEPC meeting, 7 Sept., 1999;

http://www. cern.ch/LEPHIGGS/Welcome.html.

10. A. Djouadi, J. Kalinowski and M. Spira, Comput. Phys. Commun. Res., 108 (1998) 56.

11. P. Chen, G. Horton-Smith, T. Ohgaki, A.W. Weidemann and K. Yokoya, Nucl. Instrum. Methods, A335 (1995) 107.

T. Ohgaki and T. Takahashi, Nucl. Instrum. Methods, A373 (1996) 185.

'CAIN 2.1b', K. Yokoya, see the CAIN home page, http://www-acc-theory.kek.jp/members/cain/default.html.

12. I.F. Ginzburg, G.L. Kotkin, S.L. Panfil, V.G. Serbo and V.I. Telnov, Nucl. Instrum. Methods Phys. Res., 219 (1984) 5.

V.I. Telnov, Nucl. Instrum. Methods Phys. Res., A294 (1990) 72.

D.L. Borden, D.A. Bauer and D.O. Caldwell, SLAC preprint, SLAC-PUB-5715, 1992.

13. JLC-I, KEK-Report 92-16 (1992).

14. V. Telnov, Nucl. Instrum. Methods Phys. Res., A355 (1995) 3. 


\section{Tables}

Table 1: The tree helicity amplitudes of $\gamma \gamma \rightarrow t \bar{t}$. The overall factor $4 \pi \alpha_{\mathrm{QED}} Q_{t}^{2}$ is omitted in the table.

\begin{tabular}{|c||c|c|c|c|}
\hline & $t_{R} \bar{t}_{R}$ & $t_{R} \bar{t}_{L}$ & $t_{L} \bar{t}_{R}$ & $t_{L} \bar{t}_{L}$ \\
\hline \hline$\gamma_{+} \gamma_{+}$ & $-\frac{2 m_{t}}{E_{t}} \frac{1+\beta_{t}}{1-\beta_{t}^{2} \cos ^{2} \theta_{t}}$ & 0 & 0 & $-\frac{2 m_{t}}{E_{t}} \frac{1-\beta_{t}}{1-\beta_{t}^{2} \cos ^{2} \theta_{t}}$ \\
\hline$\gamma_{+} \gamma_{-}$ & $+\frac{2 m_{t}}{E_{t}} \frac{\beta_{t} \sin ^{2} \theta_{t}}{1-\beta_{t}^{2} \cos ^{2} \theta_{t}}$ & $+2 \beta_{t} \frac{\sin \theta_{t}\left(1+\cos \theta_{t}\right)}{1-\beta_{t}^{2} \cos ^{2} \theta_{t}}$ & $-2 \beta_{t} \frac{\sin \theta_{t}\left(1-\cos \theta_{t}\right)}{1-\beta_{t}^{2} \cos ^{2} \theta_{t}}$ & $-\frac{2 m_{t}}{E_{t}} \frac{\beta_{t} \sin ^{2} \theta_{t}}{1-\beta_{t}^{2} \cos ^{2} \theta_{t}}$ \\
\hline$\gamma_{-} \gamma_{+}$ & $+\frac{2 m_{t}}{E_{t}} \frac{\beta_{t} \sin ^{2} \theta_{t}}{1-\beta_{t}^{2} \cos ^{2} \theta_{t}}$ & $-2 \beta_{t} \frac{\sin \theta_{t}\left(1-\cos _{t} \theta_{t}\right)}{1-\beta_{t}^{2} \cos ^{2} \theta_{t}}$ & $+2 \beta_{t} \frac{\sin \theta_{t}\left(1+\cos _{t}\right)}{1-\beta_{t}^{2} \cos ^{2} \theta_{t}}$ & $-\frac{2 m_{t}}{E_{t}} \frac{\beta_{t} \sin ^{2} \theta_{t}}{1-\beta_{t}^{2} \cos ^{2} \theta_{t}}$ \\
\hline$\gamma_{-} \gamma_{-}$ & $+\frac{2 m_{t}}{E_{t}} \frac{1-\beta_{t}}{1-\beta_{t}^{2} \cos ^{2} \theta_{t}}$ & 0 & 0 & $+\frac{2 m_{t}}{E_{t}} \frac{1+\beta_{t}}{1-\beta_{t}^{2} \cos ^{2} \theta_{t}}$ \\
\hline
\end{tabular}

Table 2: The masses, the total decay widths, the two-photon decay branching ratios and the $t \bar{t}$ branching ratios of $H$ and $A$ adopted in the numerical simulations.

\begin{tabular}{|c||c|c|c|c|}
\hline $\tan \beta$ & $\begin{array}{c}m_{H} \\
(\mathrm{GeV})\end{array}$ & $\begin{array}{c}\Gamma_{H} \\
(\mathrm{GeV})\end{array}$ & $\begin{array}{c}B r(H \rightarrow \gamma \gamma) \\
10^{-5}\end{array}$ & $\operatorname{Br}(H \rightarrow t \bar{t})$ \\
\hline 3.0 & 403.79 & 0.79 & 0.99 & 0.742 \\
7.0 & 400.71 & 0.50 & 0.30 & 0.207 \\
\hline \hline $\tan \beta$ & $m_{A}$ & $\Gamma_{A}$ & $B r(A \rightarrow \gamma \gamma)$ & $\operatorname{Br}(A \rightarrow t \bar{t})$ \\
& $(\mathrm{GeV})$ & $(\mathrm{GeV})$ & $10^{-5}$ & \\
\hline 3.0 & 400.00 & 1.75 & 1.53 & 0.946 \\
7.0 & 400.00 & 0.67 & 0.79 & 0.452 \\
\hline
\end{tabular}


Table 3: A rough scheme of the $\sqrt{s}_{\gamma \gamma}$ dependence of the signs of the interference terms between $\mathcal{M}_{\text {tree }}$ and $\mathcal{M}_{H, A}$. The actual switching of the sign occurs at a slightly different energy from the mass of the Higgs.

\begin{tabular}{|c|c|c|c|}
\hline & $\sqrt{s}_{\gamma \gamma}<m_{A}$ & $m_{A}<\sqrt{s}_{\gamma \gamma}<m_{H}$ & $m_{H}<\sqrt{s}_{\gamma \gamma}$ \\
\hline \hline$\Delta \sigma_{A-t r e e}^{++R R}$ & + & - & - \\
$\Delta \sigma_{H \text {-tree }}^{++R}$ & + & + & - \\
\hline$\Delta \sigma_{A \text {-tre }}^{++L} L$ & + & - & - \\
$\Delta \sigma_{H \text {-tree }}^{++L} L$ & - & - & + \\
\hline
\end{tabular}

Table 4: The effective cross sections and these relative errors with $1 \mathrm{fb}^{-1}$ for nine values of the collider energy at $\tan \beta=3$ and 7 . The effective continuum cross section and the statistical significance with $1 \mathrm{fb}^{-1}$ of the deviation of the cross section with the Higgs resonances from the continuum are also listed. The sign of $\hat{\mathcal{S}}$ indicates the sign of the deviation.

\begin{tabular}{|c|c||cc|cc|}
\hline $\tan \beta$ & $\begin{array}{c}\sqrt{s}_{e e} \\
(\mathrm{GeV})\end{array}$ & $\begin{array}{c}\sigma \\
(\mathrm{fb})\end{array}$ & $\hat{\mathcal{E}}(\sigma)$ & $\begin{array}{c}\sigma_{\text {tree }} \\
(\mathrm{fb})\end{array}$ & $\hat{\mathcal{S}}$ \\
\hline \hline 3.0 & 480 & 121.1 & 0.091 & 110.1 & +1.00 \\
& 490 & 141.4 & 0.084 & 122.6 & +1.58 \\
& 500 & 145.9 & 0.083 & 131.8 & +1.17 \\
& 510 & 140.9 & 0.084 & 138.6 & +0.19 \\
& 520 & 135.6 & 0.086 & 143.6 & -0.69 \\
& 530 & 134.7 & 0.086 & 147.3 & -1.09 \\
& 540 & 137.5 & 0.085 & 149.9 & -1.06 \\
& 550 & 141.5 & 0.084 & 151.5 & -0.84 \\
& 560 & 144.6 & 0.083 & 152.4 & -0.65 \\
\hline 7.0 & 480 & 112.0 & 0.094 & 110.1 & +0.18 \\
& 490 & 125.0 & 0.089 & 122.6 & +0.21 \\
& 500 & 131.8 & 0.087 & 131.8 & +0.00 \\
& 510 & 136.1 & 0.086 & 138.6 & -0.21 \\
& 520 & 140.0 & 0.085 & 143.6 & -0.30 \\
& 530 & 143.9 & 0.083 & 147.3 & -0.29 \\
& 540 & 147.3 & 0.082 & 149.9 & -0.22 \\
& 550 & 149.7 & 0.082 & 151.5 & -0.15 \\
& 560 & 151.1 & 0.081 & 152.4 & -0.11 \\
\hline
\end{tabular}


Table 5: The effective cross sections $\sigma^{R R}, \sigma^{L L}, \sigma^{R L+L R}, \sigma_{F}, \sigma_{B}$. The decay fractions in forward $r_{F}$ and backward $r_{B}$ of the right-handed top quark are assumed to be 0.40 and 0.60 , respectively.

\begin{tabular}{|c|c||rrc|cc|}
\hline $\tan \beta$ & $\begin{array}{c}\sqrt{s}_{e e} \\
(\mathrm{GeV})\end{array}$ & $\begin{array}{r}\sigma^{R R} \\
(\mathrm{fb})\end{array}$ & $\begin{array}{r}\sigma^{L L} \\
(\mathrm{fb})\end{array}$ & $\begin{array}{c}\sigma^{R L+L R} \\
(\mathrm{fb})\end{array}$ & $\begin{array}{c}\sigma_{F} \\
(\mathrm{fb})\end{array}$ & $\begin{array}{c}\sigma_{B} \\
(\mathrm{fb})\end{array}$ \\
\hline \hline 3.0 & 480 & 99.1 & 21.9 & 0.1 & 23.7 & 39.4 \\
& 490 & 117.0 & 24.2 & 0.2 & 27.4 & 46.2 \\
& 500 & 120.4 & 25.2 & 0.3 & 28.3 & 47.6 \\
& 510 & 117.6 & 22.9 & 0.4 & 27.1 & 46.3 \\
& 520 & 116.4 & 18.7 & 0.5 & 25.4 & 45.2 \\
& 530 & 119.5 & 14.6 & 0.6 & 24.4 & 45.7 \\
& 540 & 125.2 & 11.6 & 0.6 & 24.2 & 47.3 \\
& 550 & 130.9 & 9.8 & 0.7 & 24.5 & 49.1 \\
& 560 & 135.1 & 8.7 & 0.8 & 24.8 & 50.5 \\
\hline 7.0 & 480 & 92.1 & 19.8 & 0.1 & 21.8 & 36.5 \\
& 490 & 105.9 & 18.9 & 0.2 & 23.7 & 41.4 \\
& 500 & 114.3 & 17.2 & 0.3 & 24.4 & 44.2 \\
& 510 & 120.4 & 15.4 & 0.4 & 24.8 & 46.1 \\
& 520 & 125.9 & 13.7 & 0.5 & 25.1 & 47.8 \\
& 530 & 131.1 & 12.2 & 0.6 & 25.4 & 49.5 \\
& 540 & 135.6 & 11.0 & 0.6 & 25.7 & 51.0 \\
& 550 & 139.0 & 9.9 & 0.7 & 25.8 & 52.1 \\
& 560 & 141.3 & 9.0 & 0.8 & 25.9 & 52.8 \\
\hline \multirow{7}{*}{7.0}
\end{tabular}


Table 6: The relative errors of the effective cross sections, $\hat{\mathcal{E}}\left(\sigma^{R R}\right)$ and $\hat{\mathcal{E}}\left(\sigma^{L L}\right)$, and these statistical significances of the deviation of the cross section with the Higgs resonances from the continuum, $\hat{\mathcal{S}}^{R R}$ and $\hat{\mathcal{S}}^{L L}$. Here we assumed $1 \mathrm{fb}^{-1}$ of the luminosity multiplied by the detection efficiency. The sign of the statistical significances indicates the sign of the deviation.

\begin{tabular}{|c|c||cc|cc|}
\hline $\tan \beta$ & $\begin{array}{c}\sqrt{s}_{e e} \\
(\mathrm{GeV})\end{array}$ & $\hat{\mathcal{E}}\left(\sigma^{R R}\right)$ & $\hat{\mathcal{E}}\left(\sigma^{L L}\right)$ & $\hat{\mathcal{S}}^{R R}$ & $\hat{\mathcal{S}}^{L L}$ \\
\hline \hline 3.0 & 480 & 0.23 & 0.87 & +0.38 & +0.13 \\
& 490 & 0.21 & 0.85 & +0.53 & +0.29 \\
& 500 & 0.21 & 0.83 & +0.23 & +0.40 \\
& 510 & 0.21 & 0.89 & -0.22 & +0.38 \\
& 520 & 0.21 & 1.05 & -0.53 & +0.25 \\
& 530 & 0.20 & 1.33 & -0.61 & +0.11 \\
& 540 & 0.20 & 1.66 & -0.52 & +0.02 \\
& 550 & 0.19 & 1.96 & -0.39 & -0.01 \\
& 560 & 0.19 & 2.21 & -0.29 & -0.02 \\
\hline 7.0 & 480 & 0.24 & 0.93 & +0.07 & +0.02 \\
& 490 & 0.22 & 1.02 & +0.08 & +0.03 \\
& 500 & 0.21 & 1.13 & -0.02 & +0.02 \\
& 510 & 0.20 & 1.28 & -0.10 & +0.00 \\
& 520 & 0.20 & 1.44 & -0.14 & -0.01 \\
& 530 & 0.19 & 1.62 & -0.13 & -0.01 \\
& 540 & 0.19 & 1.80 & -0.10 & -0.01 \\
& 550 & 0.19 & 1.99 & -0.07 & -0.01 \\
& 560 & 0.18 & 2.20 & -0.05 & -0.00 \\
\hline \multirow{7}{*}{}
\end{tabular}




\section{Figures}

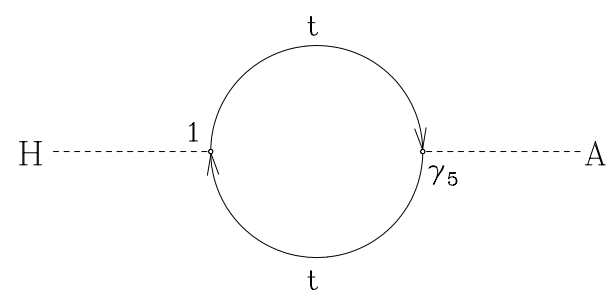

Figure 1: The top-loop amplitude that connects $H$ and $A$.

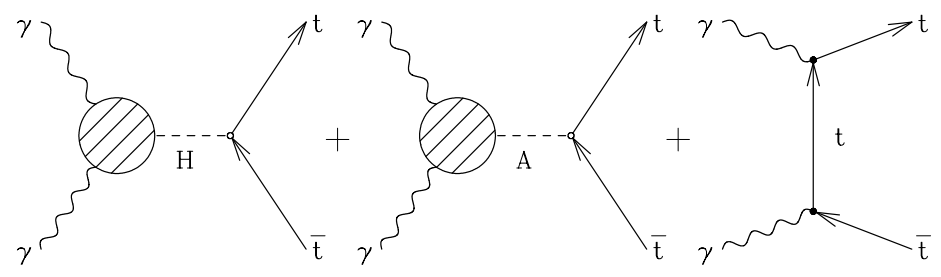

Figure 2: The dominant diagrams of the process $\gamma \gamma \rightarrow t \bar{t}$ at around the mass poles of $H$ and $A$ bosons. The u-channel tree diagram is omitted in the figure. 


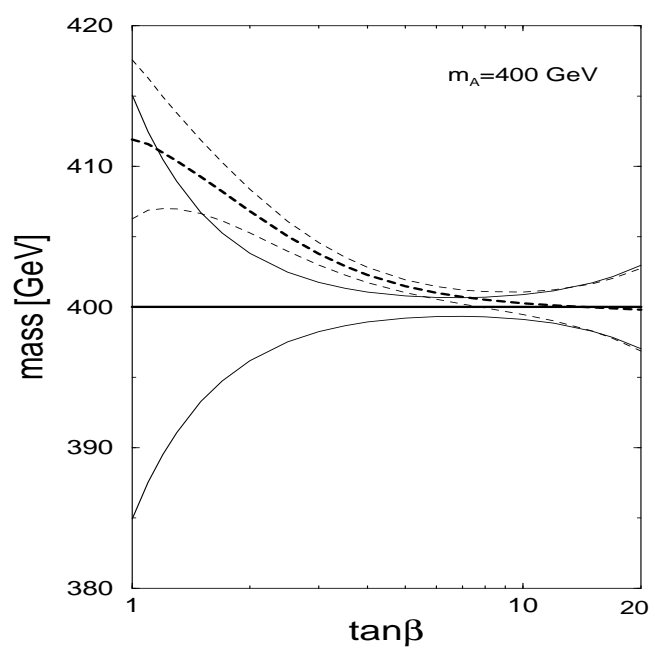

Figure 3: The masses of $H$ and $A$ are shown by bold-dashed curve and bold-solid line, respectively, as functions of $\tan \beta$. The total decay width of $H(A)$ is also indicated by the two thin-dashed (thin-solid) curves associated the mass curve, which draw $m_{H} \pm \Gamma_{H}\left(m_{A} \pm \Gamma_{A}\right)$. Here $m_{A}$ is fixed to be $400 \mathrm{GeV}$.

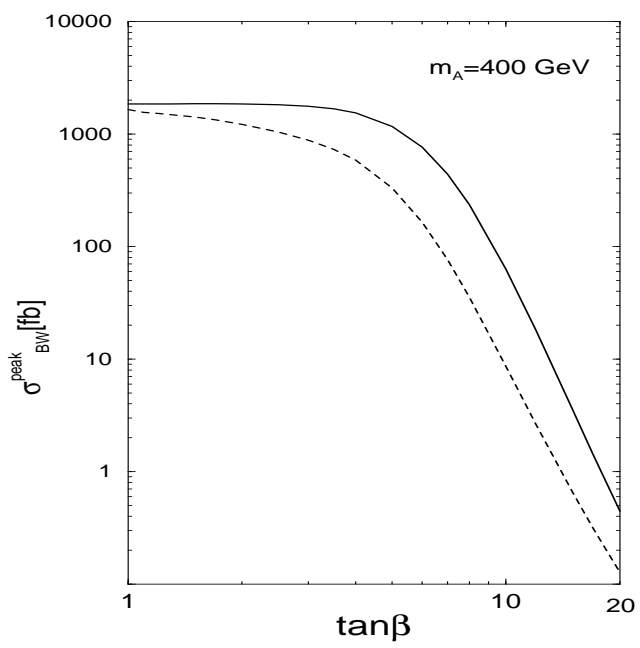

Figure 4: The peak cross sections of the process $\gamma_{+} \gamma_{+} \rightarrow H, A \rightarrow t \bar{t}$ which are estimated by the Breit-Wigner approximation. The dashed curve corresponds to $H$ and the solid curve to $A$. Note that these computations neglect either of the continuum and the interference contributions. 


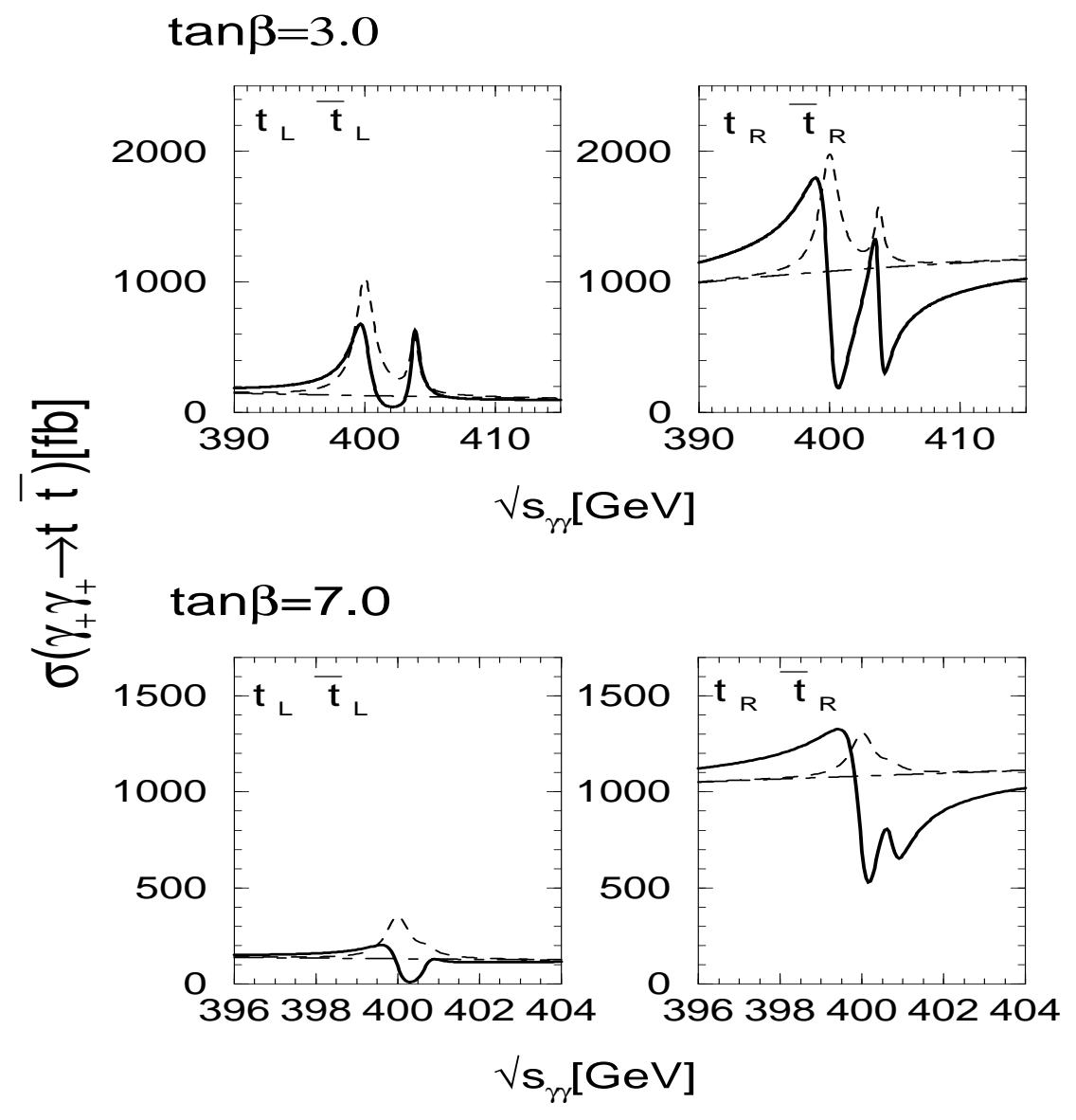

Figure 5: The cross sections of $\gamma_{+} \gamma_{+} \rightarrow t_{L} \bar{t}_{L}$ (left), $t_{R} \bar{t}_{R}$ (right) for $\tan \beta=3$ (above) and 7 (below). The solid curves show the cross sections with Higgs resonances $\sigma^{++\lambda \bar{\lambda}}$, the dashed curves the ones obtained by neglecting the interference $\sigma_{0}^{++\lambda \bar{\lambda}}$, the dot-dashed lines the continuum contributions $\sigma_{\text {tree }}^{++\lambda \bar{\lambda}}$, respectively. 


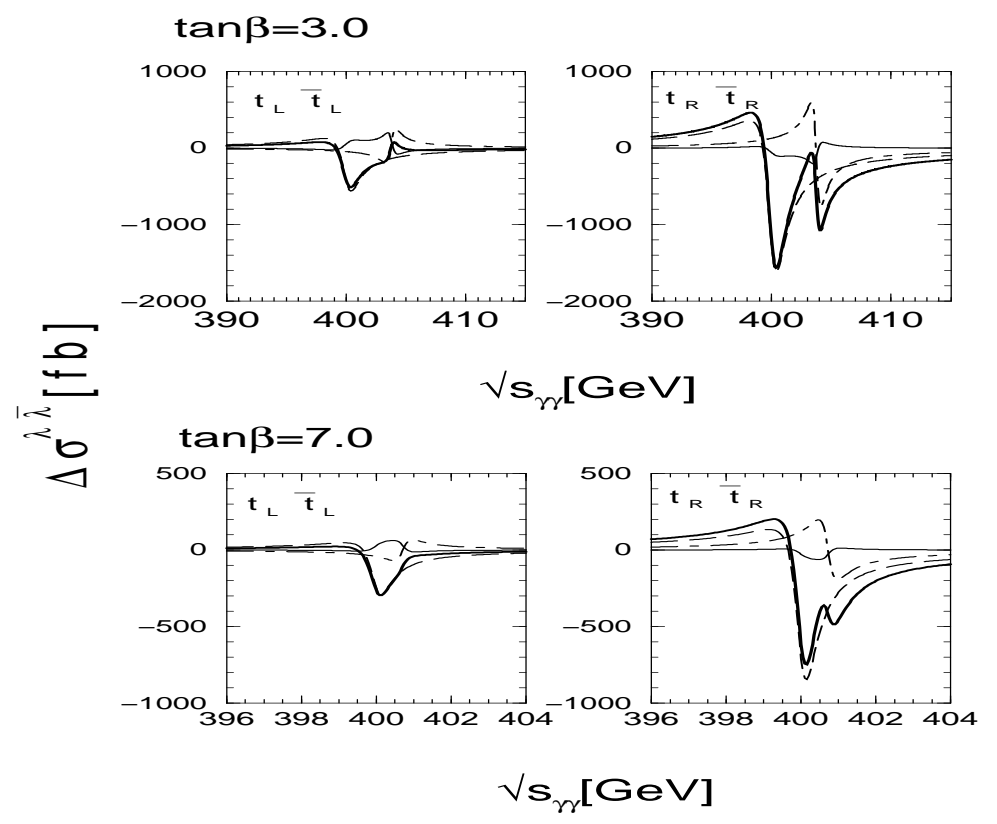

Figure 6: The interference contributions of the cross sections of $\gamma_{+} \gamma_{+} \rightarrow t_{L} \bar{t}_{L}$ (left), $t_{R} \bar{t}_{R}$ (right) for $\tan \beta=3$ (above) and 7 (below). The thin-solid, dot-dashed and dashed curves show $\Delta \sigma_{A-H}^{++\lambda \bar{\lambda}}, \Delta \sigma_{H \text {-tree }}^{++\lambda \bar{\lambda}}$ and $\Delta \sigma_{A \text {-tree }}^{++\lambda \bar{\lambda}}$, respectively, and the bold-solid ones mean the sum of these three, $\Delta \sigma^{++\lambda \bar{\lambda}}$.

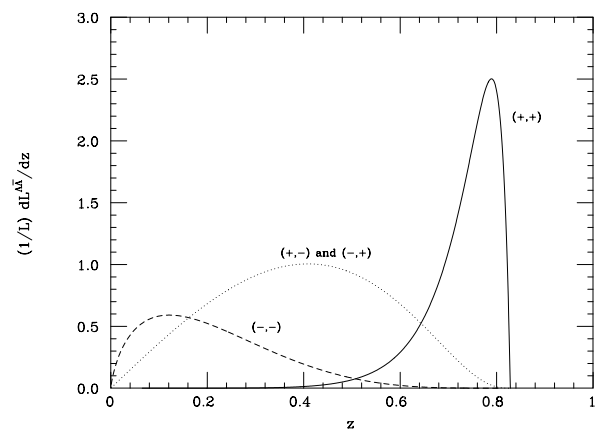

Figure 7: The luminosity distributions of the $\gamma \gamma$ colliders. The solid, dotted and dashed curves are correspond to the $\gamma \gamma$ collisions with the helicities of,+++- and -+ , and -- , respectively. The horizontal axis $z$ means the energy fraction of the colliding photons $E_{\gamma \gamma}$ to the original electron collider $\sqrt{s}_{e e}$. 


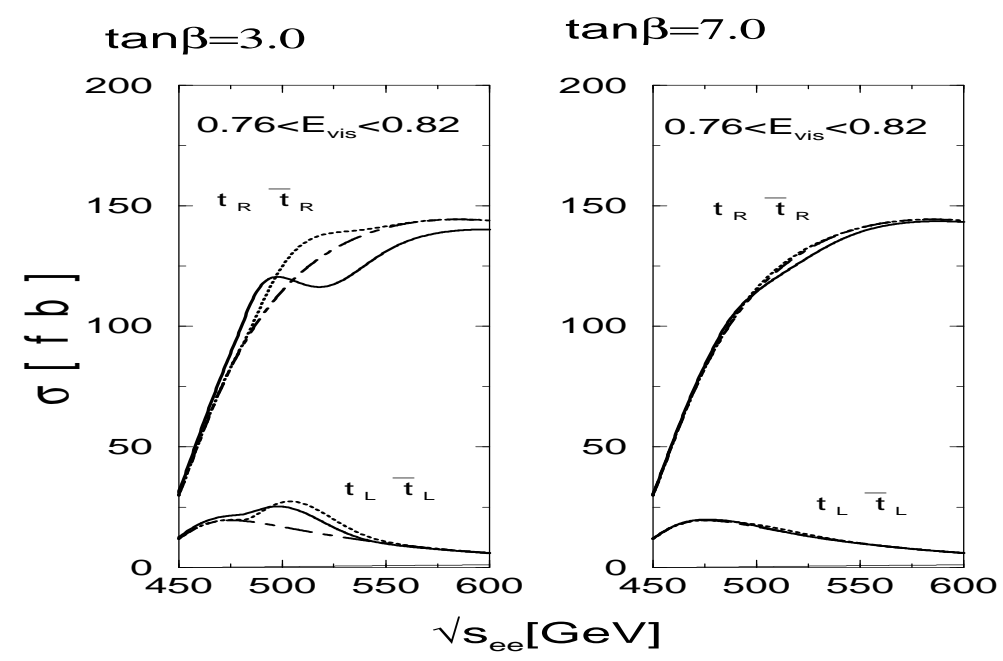

Figure 8: The effective cross sections convoluted by the $\gamma \gamma$ luminosity with the visible energy cut are illustrated. The bold-solid curves correspond to the correct cross sections $\sigma^{\lambda \bar{\lambda}}$, while dotted and dot-dashed ones to $\sigma_{0}^{\lambda \bar{\lambda}}$ and $\sigma_{\text {tree }}^{\lambda \bar{\lambda}}$, respectively. The upper curves are for $t_{R} \bar{t}_{R}$, and the lower ones for $t_{L} \bar{t}_{L}$. The sum of the tree cross sections for $t_{R} \bar{t}_{L}$ and $t_{L} \bar{t}_{R}, \sigma^{R L+L R}$, are also plotted in the thin-solid line located very near to the bottom horizontal axis. The left figure is for $\tan \beta=3$, and the right for $\tan \beta=7$. 